\title{
CYLINDRICAL ROLLER BEARING LUBRICATION REGIMES ANALYSIS AT LOW SPEED AND PURE RADIAL LOAD
}

\author{
Jakub Chmelař**, Karel Petr, Petr Mikeš, Vojtěch Dynybyl \\ Czech Technical University in Prague, Faculty of Mechanical Engineering, Technická 4, 16607 Praha 6, Czech \\ Republic \\ * corresponding author: jakub.chmelar@fs.cvut.cz
}

\begin{abstract}
.
In this article, we describe a method of the roller bearing lubrication regime prediction. It uses a mathematical model of the bearing based on the standard ISO/TS 16281 to obtain the external load distribution over the rolling elements. We consider the effects of press fit and thermal gradient between bearing rings on the internal clearance. The model is limited to an input of an uni-axial radial load and it neglects the centrifugal forces and gyroscopic moments due to its application for the region of low speeds. The lubrication film thickness is evaluated for the most loaded rolling element by a numerical solution of Reynolds' equation for the line contact. The assessment of the lubrication regime takes into the account the surface roughness by employing the lubrication coefficient as an output parameter. Presented outcomes of the study, based on the measured geometry of the bearing, show the importance of an appropriate lubricant selection for the application.
\end{abstract}

KEYWORDS: Bearing, lubrication, elastohydrodynamic, load distribution, Reynolds.

\section{INTRODUCTION}

The importance of the rolling element bearing lubrication is indispensable. The lubricant minimizes the friction between rolling elements and races and greatly contributes to the heat removal during the course. Modern lubricants are formulated with additives that help to protect interacting surfaces in the local challenging conditions of contacts. The selection of a lubricant vastly depends on the desired application and its operating conditions respectively. Heavily loaded industrial gearbox bearings will be lubricated by an oil with different properties than high speed bearings of machine tool spindles in order to achieve optimal operating conditions.

A guideline for an estimation of the oil viscosity with respect to the bearing size, operating speed and temperature is provided in the standard ISO 281 [1]. The driving parameter is the $\kappa$ coefficient, that is used to select the life modification factor $a_{i s o}$. The calculation takes the bearing load into the account during the assessment of $a_{i s o}$, not the lubrication factor itself. The method is structured to be used by engineers, but simplification does not enable the insight into the nature of the lubrication film formation. A more detailed method for the estimation of the lubrication film thickness is based on semi-analytical equations that were derived by approximating results from a numerical solution of the Reynolds' equation. The equations for basic types of Hertz contacts (e.g. elliptical, line) are presented in [2, 3] or [4. The limitation of such approach is that the range of inputs for that it returns a reasonable result is usually unknown. Only one paper was found to deal with the relevancy [5], where author concludes that most of the analysed equations tend to over-predict the film thickness, so such equations should be taken with care.

To obtain the load distribution in the radial rolling element bearing, Stribeck [6] derived series of simple analytical equations that take into account a rolling element complement, contact angle and a radial force. Equations were derived for a standard bearing clearance. Since the bearing stiffness is non-linear, the equations are valid only for a small range of conditions. For research purposes, the results might be misleading. The only way to obtain correct values of a load distribution is by solving the load distribution with respect to the radial clearance and stiffness of elements. An example of such method is described in the standard ISO/TS 16281 [7].

A method described in this paper combines the numerical solution of Reynolds equation published by Venner [8 and the bearing load calculation as described in the ISO/TS standard [7]. It provides a toolbox enabling to study the effect of parameters, such as external load, running speed, press fit of inner ring, temperature gradient or lubricant properties, on lubrication regimes during the operation of a bearing.

The method is employed to support the research activities focused on bearing monitoring by providing lubrication states predictions.

\section{Components of BeARING ANALYSIS METHOD}

\subsection{MODEL OF BEARING}

The bearing model stands on the cornerstone of the method for the prediction of the modified reference rating life for universally loaded bearings described in 


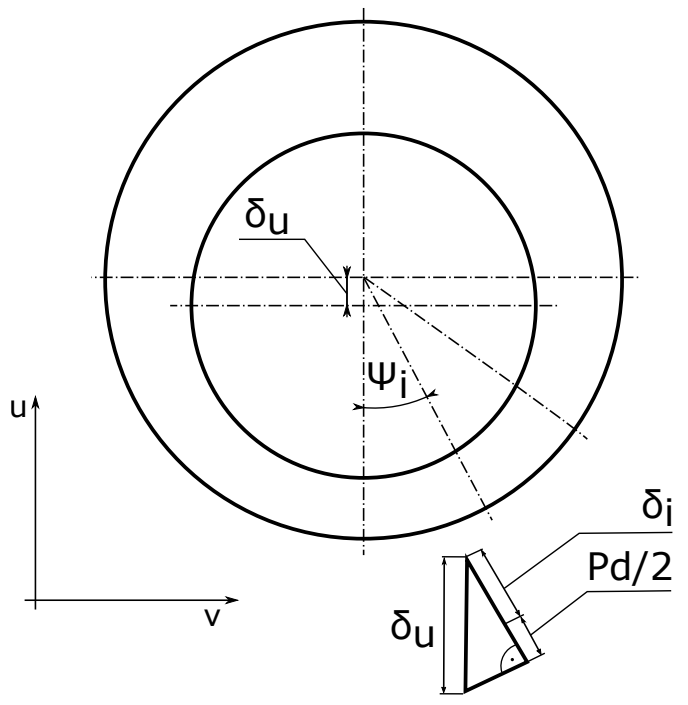

FiguRE 1. Bearing coordinates and the displacement concept scheme.

the standard [7]. It allows a calculation of an external load distribution over rolling elements taking into the account the inner bearing geometry such as the diameter of both races and the size of rolling elements. Following assumptions are accepted when applying the model:

- small displacements relatively to the size of bearing

- linear behaviour

- low rotation speed - insignificant centrifugal forces on the rollers

- load acts in a radial direction only

To calculate the load distribution a load-deflection relation for the roller and raceway contact is defined. For a single roller it is per (1).

$$
Q_{i}=K_{b} \cdot \delta_{i}^{10 / 9}
$$

where the stiffness constant $K_{b}$ is for the roller - raceway contact expressed as a function of the total roller length and a stiffness constant per (2).

$$
K_{b}=c \cdot L_{w e}^{8 / 9}
$$

Published values of the stiffness constant $c$ for a roller with two raceways contacts vary across the literature. For example, in [6], the value 80,600 is provided for a single sided contact of steel roller with a steel raceway, that results in 40, 300 for a two-sided contact. In the standard [7, a lower value of 35,948 is used, which might account for a specific roller geometry modification, discussed later in the text.

The displacement of the inner ring under pure radial load acting in the negative direction of the coordinate $u$ per Figure 1 is designated by $\delta_{u}$. The projection of the displacement at the position of each rolling element is expressed by the first member of (3). To isolate the displacement contribution by compliance of elements in contacts, a radial clearance $P_{d} / 2$ is subsequently subtracted from the displacement of each rolling element in the second member of (3). If the resulting displacement of element is negative, then the zero substitutes the result as no contact happens.

$$
\begin{aligned}
& \delta_{i} \geq 0: \delta_{i}=\delta_{u} \cdot \cos \left(\psi_{i}\right)-\frac{P_{d}}{2} \\
& \delta_{i}<0: \delta_{i}=0
\end{aligned}
$$

The total force supported by a rolling element is obtained by substituting (3) and (2) (1).

To account for a roller longitudinal shape modification, generally used to smooth the contact stress distribution and avoid edge loading, the standard [7] uses a lamina approach. It splits the roller to a finite number of sections (laminas), each with an assigned equal stiffness value, corresponding to the number of slices. For each slice, the (4) expresses its displacement under the load. The negative value of the deformation - loss of contact - is treated as null.

$$
\begin{aligned}
& \delta_{i, j} \geq 0: \delta_{i, j}=\delta_{i}-2 \cdot P_{j} \\
& \delta_{i, j}<0: \delta_{i, j}=0
\end{aligned}
$$

There are various profile modifications proposed in the literature 6]. The derivation of an optimal profile based on a mathematical analysis is published in 9. Its applicability is, however, limited due to manufacturing difficulties resulting from a precision grounding of the shape. An example of a widely used two-parameter cylindrical modification profile per (5) [10]. is depicted in Figure 22 The defining parameters are the radius of the roller end $R$ and the length coordinate of the cylindrical section $A$.

$$
\begin{aligned}
& y_{j}<|d|: P_{j}=0 \\
& y_{j}>|d|: P_{j}=R-\sqrt{R^{2}-\left(y_{j} \pm A\right)^{2}}
\end{aligned}
$$

The external load distribution over the rolling elements is obtained by a solution of a force-balance expressed by (6). It is created by combining equations 2 and 5 into one. The equation is solved for unknown $\delta_{u}$ so that it minimizes. In the current study, a zerocrossing searching function fzero implemented in $G N U$ Octave [11] was used to get the result.

$$
F-\sum_{i=1}^{Z}\left(\sum_{j=1}^{n_{s}}\left(\delta_{i, j}^{10 / 9} \cdot \frac{K_{b}}{n_{s}}\right) \cdot \cos \left(\psi_{i}\right)\right)=0
$$

The internal radial clearance has a significant impact on the load distribution over the elements. Virtually no bearing operates with the nominal clearance with which it leaves the production. The radial clearance is reduced due to either the effect of bearing inner 


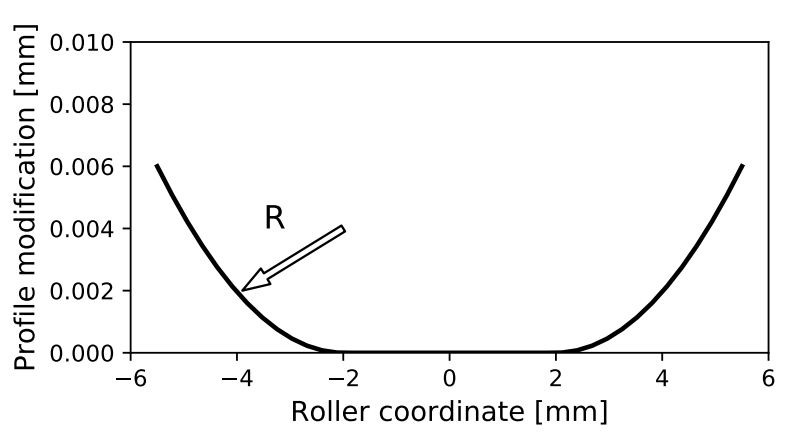

Figure 2. The roller cylindrical profile of transversal modification per (5)

ring press fit on the shaft or the temperature gradient between the inner and outer ring during the operation. The compliance of the housing and a localized point loading of the bearing also has its contribution, but it is difficult to estimate its effect without a structural analysis of the supporting housing by means of FEM. Therefore, such effects were neglected in this study.

An initial radial clearance reduction by the press fit of the inner ring on the shaft is modelled by employing the open thick walled cylinder theory. The radial displacement of the inner race is expressed for the interference fit $I$ per (7) [6]

$$
\begin{aligned}
F & =\frac{d_{i r}}{d_{s}} \\
\delta_{d i r} & =\frac{2 \cdot I \cdot F}{\left[F^{2}-1\right]\left\{\left[\frac{F^{2}+1}{F^{2}-1}+\frac{1}{\xi_{i r}}\right]+\frac{E_{i r}}{E_{s}}\left[\frac{F^{2}+1}{F^{2}-1}-\frac{1}{\xi_{s}}\right]\right\}}
\end{aligned}
$$

The heat removal from the outer race by the convection is generally more effective than from the inner race. As a result, the temperature gradient arises between the inner and outer ring. A linear temperature gradient between the inner race and rollers with respect to the outer race is considered in the current model. The expression for the calculation of the radial clearance accounting for the gradient and press fit inner ring displacement is stated in (8).

$$
\begin{aligned}
P d & =D_{o r}-\left(d_{i r}+\delta_{d i r}\right)\left(1+\alpha_{T} \Delta T\right)- \\
& -2 D_{w}\left(1+\alpha_{T} \frac{\Delta T}{2}\right)
\end{aligned}
$$

The operating radial clearance could be then used for the calculation of the load distribution per (3).

The described lamina model is used to obtain a contact pressure distribution along the profile of the roller. For each strip, the corresponding partial load is calculated per $(9)$.

$$
f_{i, j}=\delta_{i, j}^{10 / 9} \cdot \frac{K}{n_{s}}
$$

The Hertz theory of the line contact is subsequently employed to obtain the contact semi-width for each strip per 10 and the contact stress per (11).

$$
\begin{gathered}
b_{i, j}=\sqrt{\frac{8 f_{i, j} R^{\prime}}{\pi \cdot E^{\prime}}} \\
p_{i, j, k}=\sqrt{1-\frac{x_{k}^{2}}{b_{i, j}^{2}}}
\end{gathered}
$$

The accuracy of this approach is, however, limited to the cylindrical section of the roller, where results of the contact stress are precise. Close to the ends, where rollers are rounded, the results diverge from the exact solution. A proposed effective approach to numerically solve the contact of rollers with the arbitrary geometry was published in [12], but not implemented in this study.

\subsection{BeARING Kinematics}

For the subsequent lubrication analysis, the speed of both bodies entering the contact - also referred as the entraining speed, is a very important parameter. For a bearing whose operating speed of the inner ring is known, the entraining speed is calculated using the following 12 . For the derivation, the reader is cross-referred to $[2]$.

$$
u s=\frac{d_{i r} \omega_{i r}}{4} \cdot \frac{d_{i r}+2 D_{w}}{d_{i r}+D_{w}}
$$

\subsection{MODEL OF LUBRICATION LAYER}

The Elastohydrodynamic Lubrication (EHL) film model is the second component of the bearing analysis. It uses results of the bearing model to obtain inputs such as loads, roller and race geometry, and their material parameters.

Reynolds' equation for the line contact - 13 is used to model the lubrication film in the centre section of the roller. It was derived from the general NavierStokes equations and describes the flow of viscous, compressible, Newtonian fluid through a thin orifice. The following assumptions are accepted, to use the equation for a roller-race contact:

- The cylindrical section of a roller is considerably long.

- The fluid enters and exits the contact in perpendicular direction to the roller axis.

- The speed of the liquid is constant.

- The flow rate is neglected in an axial and vertical direction.

- Load, speed and temperature of the fluid are time independent.

- Isothermal conditions are satisfied within the fluid

- The contact is fully flooded - no starvation.

- Both surfaces are smooth 
The right hand side of the 13 describes the flow dependency on the shape of the orifice in the direction of flow, also referred to as the wedge effect, the left hand side then describes the flow due to the pressure gradient.

$$
\frac{\partial}{\partial x}\left(\frac{\rho h^{3}}{\eta} \frac{\partial p}{\partial x}\right)=6 u_{s} \frac{\partial(\rho h)}{\partial x}
$$

The film thickness in the analysed section is described per (14) by the central constant film thickness $h c$ with a superimposed shape of contact bodies and their deformation $\delta(x)$. The $h c$ is adjusted with respect to the force balance equation as an integration constant during the calculation.

$$
h(x)=h c+\frac{x^{2}}{2 R^{\prime}}+\delta(x)
$$

The deformation of the contact bodies by an applied contact pressure $p(x)$ in the integral form is per 15

$$
\delta(x)=\frac{4}{\pi E^{\prime}} \int_{-\infty}^{\infty} \ln \left|\frac{x-x^{\prime}}{x_{0}}\right| p\left(x^{\prime}\right) d x^{\prime}
$$

The force balance equation 16 must be valid to obtain reasonable results. It might also be used as one of the convergence criteria.

$$
f=\int_{-\infty}^{\infty} p(x) d x
$$

Rhoeland's expression 17 describes the effect of the pressure in the liquid on the dynamic viscosity. It plays a significant role in the ability of fluid to form the lubrication film and transfer loads. The expression is widely used in models, because of its good accuracy within a wide range of loads. [13].

$$
\eta=\eta_{0} e^{\left\{\ln \left(\frac{\eta_{0}}{\eta_{R}}\right)\left(\left(1+\frac{p}{p_{R_{1}}}\right)^{z}-1\right)\right\}}
$$

Where the Rhoeland's coefficient $z$ is per $(18)$.

$$
z=\frac{\alpha p_{R 1}}{\ln \left(\frac{\eta_{0}}{\eta_{R}}\right)}
$$

The compressibility of the fluid is described by another widely used equation 19 .

$$
\rho=\rho_{0} \frac{p_{R 2}+1.34 p}{p_{R 2}+p}
$$

The lubrication film model is approximated by using the finite difference method and solved at a 1D domain by the Newton-Raphson numerical technique. The solution follows the approach for heavily loaded
EHL contacts described by Venner in 8. To make the non-linear problem with decent gradients numerically stable, even for high-contact pressures, and to accelerate the convergence, the multigrid solution method is employed. For a comprehensive description, the reader is kindly advised to see the respective literature or 14. In the current article, the description is limited only to a physical background to establish the understanding.

\subsection{LUBRICATION REGIME ASSESSMENT}

The assessment of the lubrication regimes is performed by the lubrication coefficient $\lambda$ that is defined as the ratio of the minimal thickness of the lubrication film to a composite roughness of both contact bodies. 4

$$
\lambda=\frac{h_{0}}{\sqrt{R q_{\text {race }}^{2}+R q_{\text {roller }}^{2}}}
$$

The 2D amplitude roughness parameter $\mathrm{Rq}$ is used in the study. Under the assumption that the surface roughness follows Gaussian distribution, one may use the relationship between the commonly used arithmetic mean value - Ra and the RMS - Rq: $R q=1.25 \times R a$. If the spatial $-3 \mathrm{D}$ parameter $S q$ [15] of the surface roughness is available, one may use it instead of the $2 \mathrm{D}$ and achieve a more accurate prediction. The spatial parameters better characterize the overall state of the surface.

Lubrication regimes are distinguished by the $\lambda$ ratio that is as follows [16]:

- $\lambda<1$ : Boundary friction regime

- $1<\lambda>3$ : Mixed friction regime

- $\lambda>3$ : Elastohydrodynamic friction regime

The transition between regimes is not sudden but continuous, therefore, the values should rather be considered as indicators.

\subsection{BEARING MEASUREMENT}

Details of the bearing's internal geometry is a proprietary information of every manufacturer. To perform a comprehensive analysis of a bearing, the means of modern metrology were used. Geometry of the bearing rings (bore, external diameter and diameter of races) and rolling elements (diameter and profile modification) were measured on a Prismo coordinate machine by Carl Zeiss. Unlike conventional contact measurement machines that measure the object by a small number of discrete points, the Prismo leverages a measurement technology using a high density of measured points enabling a considerable reduction of data scatter and improvement of the measurement accuracy. Recorded data are filtered and approximated by selected evaluation methods. To accurately capture the bearing ring dimension, a circular scanning path of probe was set. The data were recorded with a frequency of $0.1 \mathrm{~mm}$ along the profile at a probe 


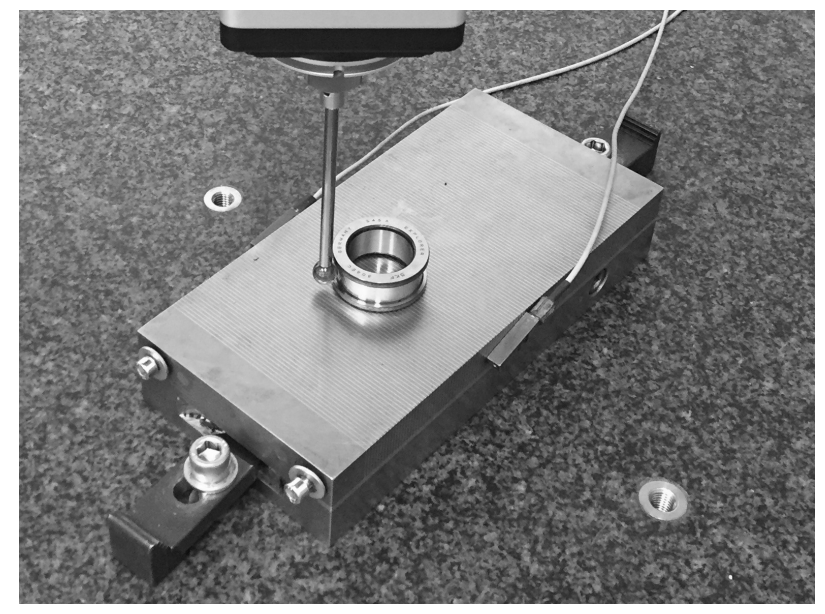

FIGURE 3. Inner race geometry measurement.

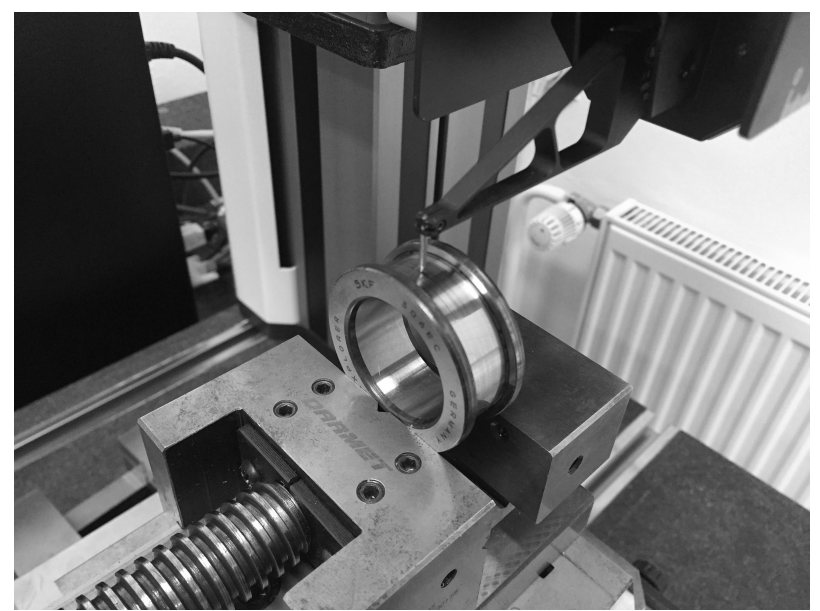

FIGURE 4. Inner race surface texture measurement.

motion speed of $5 \mathrm{~mm} / \mathrm{s}$ and evaluated using a low pass filter with $50 U P R$. In case of the roller profile measurement, higher data density was needed to capture the micro-geometry. The probe motion speed was $1 \mathrm{~mm} / \mathrm{s}$, data were recorded with the frequency of $0.01 \mathrm{~mm}$ and a low pass spline filter with a length of $0.8 \mathrm{~mm}$ was used. To mitigate the risk of bearing rings deformation due to clamping, a magnetic table was used as depicted in Figure 3

The surface roughness of both races and rollers were measured on a combined contour and roughness measurement machine MarSurf LD 120 by Mahr. The Fig. 4 depicts the clamped inner ring in the vices and the probe touching the race. Two 2D amplitude parameters of surface roughness were obtained: the Arithmetic Mean Height - Ra and the Maximum Profile Height - Rz. The evaluated profile was $1.75 \mathrm{~mm}$ long, with a sampling of $1 \mu \mathrm{m}$, oriented in the axial direction of the bearing. The measurement was performed at three spots on the race and on three rollers. For each bearing component, the stated surface roughness value is the mean of the measured $\mathrm{Ra}$ values.

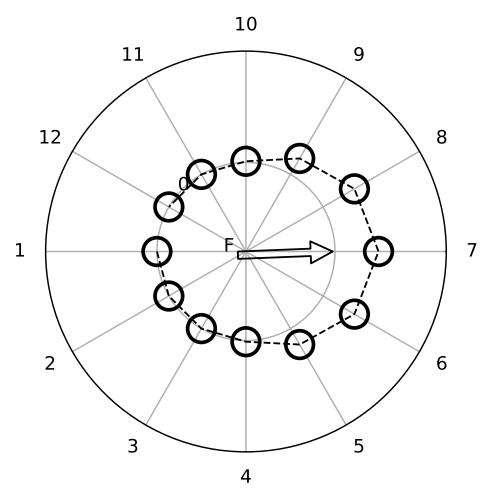

FigURE 5. Designation of rolling elements, external load force vector and the example of the external load distribution over elements.

Selected measured parameters are listed in Table 1. The processed and parameterized roller profile modification is depicted in Figure 2

\begin{tabular}{lll}
\hline Measured par. & Value & Unit \\
\hline$D w$ & 10.984 & $\mathrm{~mm}$ \\
Lwe & 11 & $\mathrm{~mm}$ \\
$R a_{i r}$ & 0.040 & $\mu \mathrm{m}$ \\
$R a_{D w}$ & 0.024 & $\mu \mathrm{m}$ \\
$Z$ & 12 & - \\
$R$ & 1020 & $\mathrm{~mm}$ \\
$A$ & 2 & $\mathrm{~mm}$ \\
\hline Calculated par. & Value & Unit \\
\hline$P C D$ & 51.525 & $\mathrm{~mm}$ \\
$P d$ & 0.034 & $\mathrm{~mm}$ \\
\hline
\end{tabular}

TABLE 1. Actual measured values of bearing parameters.

\section{Results And Discussions}

The model described in the previous section is used for the study of a roller load distribution sensitivity on the variation of the bearing geometrical and operational parameters. The effect of parameters is assessed independently, using nominal values of remaining inputs per Table 2 Subsequently, the thickness of the lubrication film is calculated and studied for the most loaded element using the outputs from the bearing model. A design space of parameters used for the analysis comprises a range of operating speeds, loads and lubricant properties.

Depicted figures with results of analyses use the designation of rolling elements corresponding to Figure 5. The external load is always considered acting on the inner ring, in the direction of the element No. 7.

\subsection{LOAD DISTRIBUTION}

The geometrical parameter that drives the load distribution is the radial clearance [6]. Each bearing is 


\begin{tabular}{lcc}
\hline Parameter & Value & Unit \\
\hline$F$ & $8775(\approx 0.15 C)$ & $N$ \\
$n$ & 500 & $r p m$ \\
$P d_{0}$ & 0.034 & $m m$ \\
$\Delta T$ & 0 & ${ }^{\circ} \mathrm{C}$ \\
$I$ & 0.012 & $\mathrm{~mm}$ \\
$E$ & $2.1 E+5$ & $\mathrm{~N} / \mathrm{mm}^{2}$ \\
$\xi$ & 0.3 & - \\
$\alpha_{T}$ & $11.4 E-6$ & - \\
\hline
\end{tabular}

TABLE 2. Nominal input parameters used in the study.

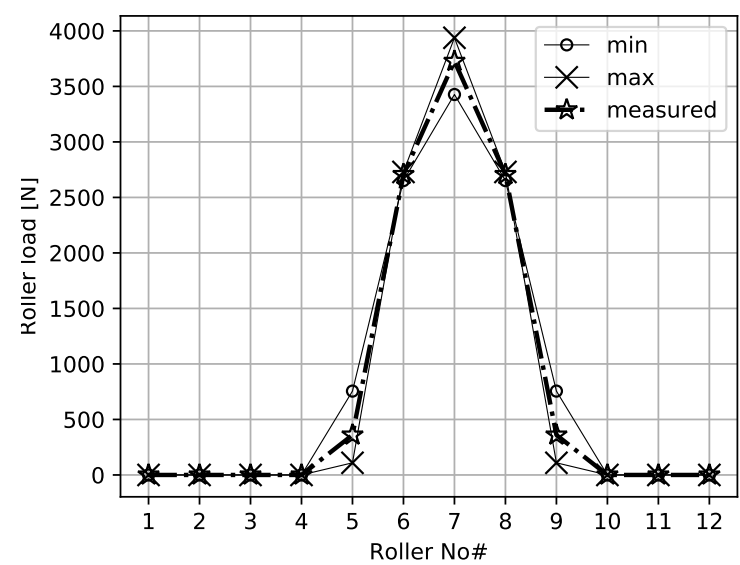

FIGURE 6. Effect of radial clearance on the roller load.

assembled with the initial radial clearance, that is stated in the standard ISO 5732 [17] for unmounted bearings. During operation, the resulting fit of the inner race on the shaft or the temperature gradient between rings makes it (temporarily) change and it becomes the operational radial clearance.

An initial study was conducted to obtain the effect of the initial clearance variation on the roller load distribution. Results are depicted in Figure 6 6 It shows the loading of each element, when the nominal bearing load is acting in the direction of the roller No. 7. The min and max clearance respectively corresponds to the boundary values per standard for analysed bearing it is in the range from $20 \mu \mathrm{m}$ to $45 \mu \mathrm{m}$. A curve designated as measured applies for the clearance value obtained from results of the bearing geometry measurement.

The result of a subsequent study, where the interference fit of the bearing inner race on the shaft is varied, is in Figure 7. The solid shaft is considered to be manufactured in the tolerance class $m 5: e i=0.008 \mathrm{~mm}$,es $=0.017 \mathrm{~mm}$. Tolerance values of the bearing bore correspond to the ISO 492 [18]: $E S=0, E I=-0.01$. The measured case was calculated using the measured bearing bore diameter. The value of the shaft diameter tolerance for that case was estimated as upper quartile - $Q 3$ of the tolerance

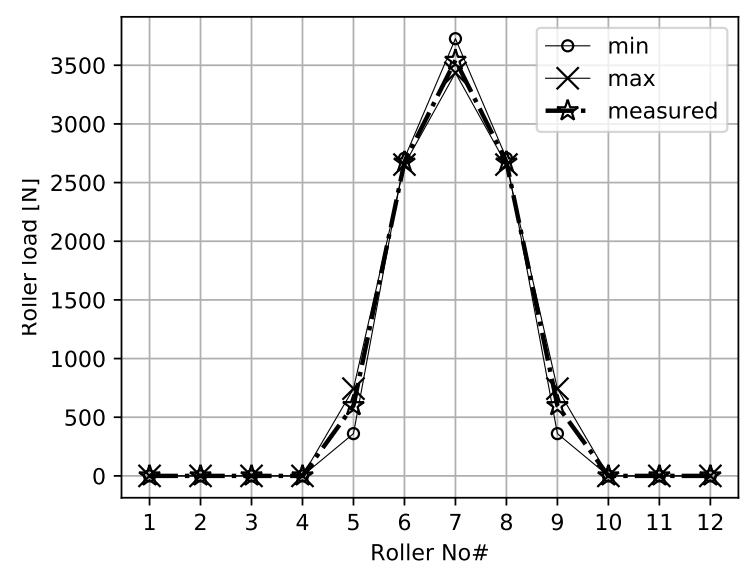

FiguRE 7. Effect of interference fit on the roller load.

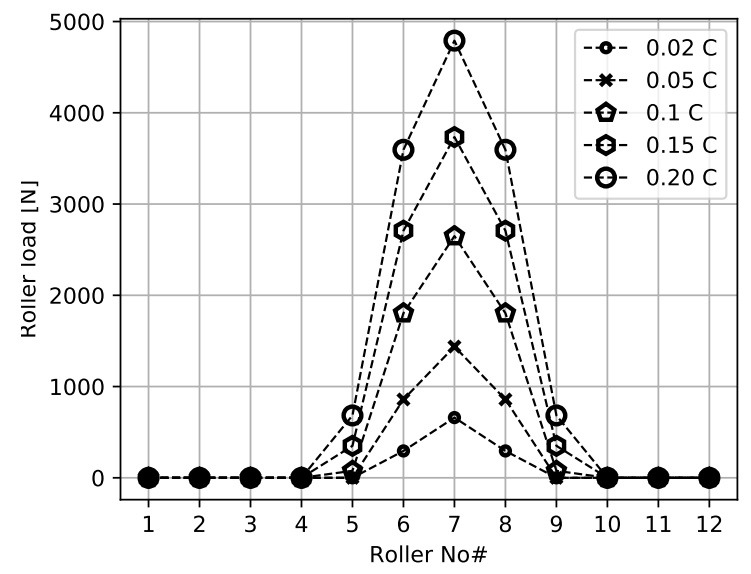

Figure 8. Effect of external load magnitude on the roller load.

field for the class. The resulting interference fit than yields in: $0.020 \mathrm{~mm}$.

It is obvious that the interference fit for the considered tolerance class and current bearing has a lower effect on the load distribution when compared to the effect of the clearance variation depicted 6 , the bearing could be assembled with. The tolerance of the initial clearance results in $500 \mathrm{~N}$ or $12 \%$ load difference for the most loaded element.

The effect of the external radial load on its distribution over the rollers is depicted in the Figure 8. The external load magnitude is expressed as a fraction of the bearing dynamic capacity $\mathrm{C}$, varying from the recommended minimal operational load $0.02 C$ up to $0.2 C$, that is considered as a heavy load $[6$ for this bearing type.

The temperature gradient between the bearing rings is another operational parameter that influences the operational radial clearance. The reasonable value of the gradient for the current bearing was set from $5^{\circ} \mathrm{C}$ to $20^{\circ} \mathrm{C}$. Results of the analysis are depicted in the Figure 9 It clearly shows that within the gradient 


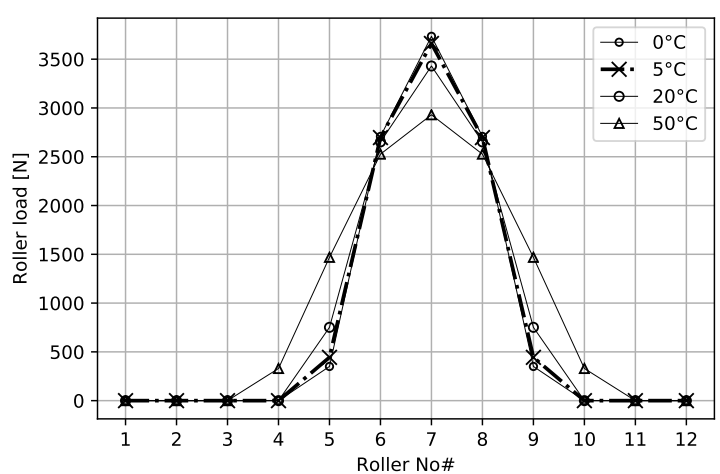

FiguRE 9. Effect of temperature gradient $\Delta T$ on the roller load.

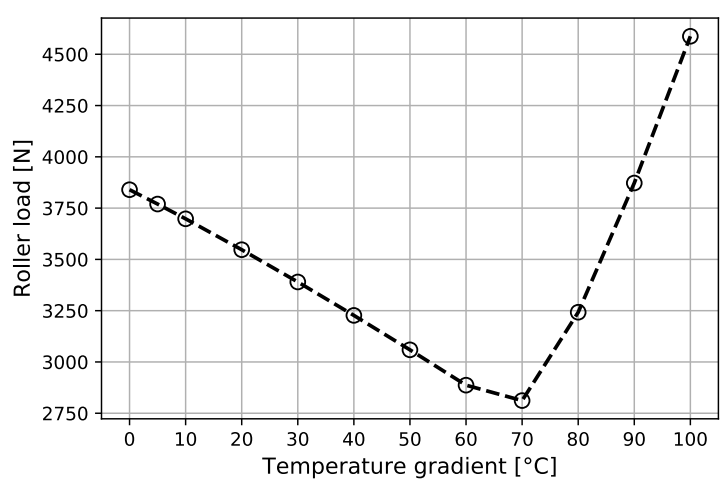

Figure 10. Effect of temperature gradient $\Delta T$ on the most loaded rolling element - No.7.

up to $20^{\circ} \mathrm{C}$, the load distribution is not significantly influenced, however, when the gradient is increased up to $50^{\circ} \mathrm{C}$, the clearance becomes delimited, resulting in the load distribution acting in a range of $180^{\circ}$. The distribution of the load over more rollers causes unloading the maximally loaded element, however, when the temperature gradient is further increased, the load steeply increases as depicted in Figure 10. This phenomenon is especially important if the bearing has a reduced internal clearance, e.g. precision bearings of machine tools.

\subsection{LUBRICATION FILM THICKNESS AND REGIME}

The formation of the lubrication film in the contact depends on multiple factors. The entraining speed of bodies into contact, applied load and lubricant's property such as actual dynamic viscosity belong among the most important ones. The sensitivity study of a minimal lubrication film thickness on the named parameters was carried out in this paper and the results are presented hereinafter.

The study was performed for the bearing operating under the common range of conditions. It is lubricated by the bearing oils listed in the Table 3. All oils are mineral, petroleum based, formulated only
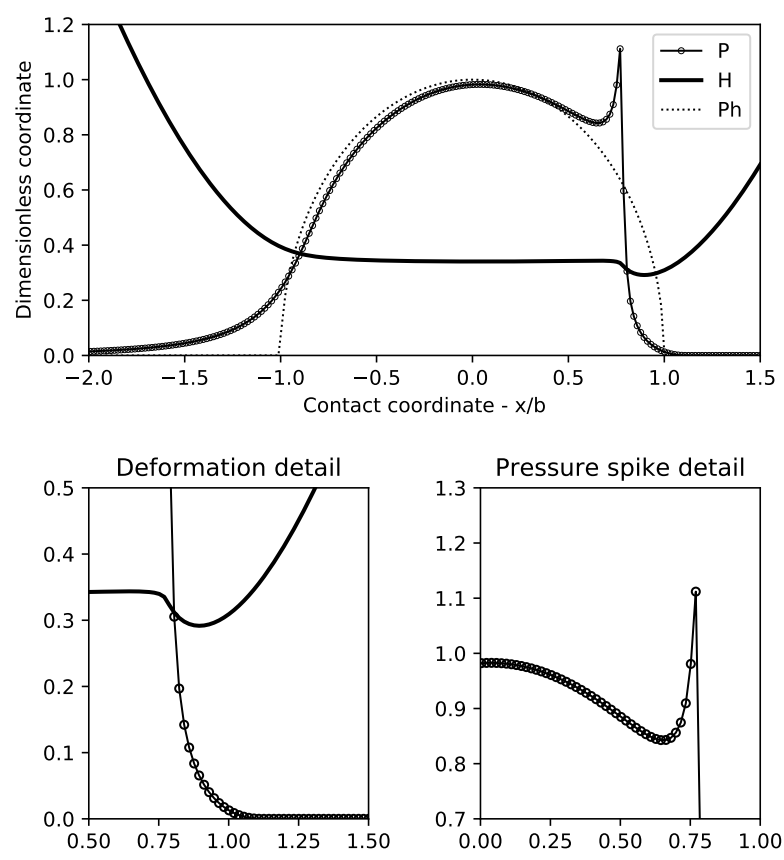

Figure 11. Contact pressure and lubrication film thickness distribution.

with additives preventing the degradation of the lubricant by oxidation. It is assumed that the lubricant's temperature is held at a constant level of $40^{\circ} \mathrm{C}$ in the contact and the lubricant supply is sufficient to produce the fully flooded contact, so no starvation occurs.

The Figure 11 shows a typical pressure distribution in the line contact with a fully developed EHL film. It basically copies the typical Hertz contact distribution - in the figure depicted by a dotted line, beside the inlet and exit. Close to the contact inlet, the pressure smoothly develops from the environmental to maximal value. On the other side, in the vicinity of the contact exit, there is an apparent pressure spike followed by a dramatic pressure gradient back to the ambient pressure. The spike is a result of the contact bodies' deformation and hydrodynamic pressure forces within the compressed lubricant. Its shape depends on the operating conditions and lubricant's parameters. The minimal film thickness is measured at the location of the pressure spike, where the mutual deformation of bodies forms a shoulder and surfaces approach closest to each other.

The Figure 12 shows curves of the lubrication parameter $\lambda$ for different properties of the lubricant per Table 3 and operating conditions of the bearing. The speed of the bearing varies from $500 \mathrm{rpm}$ to $3000 \mathrm{rpm}$, pair of curves for each lubricant are boundaries obtained by a variation of the applied bearing load from $0.02 C$ to $0.2 C$. By comparing the curves it is possible to read the sensitivity of the parameter $\lambda$ on operating parameters varied in this study. Following findings could be drawn: 


\begin{tabular}{llccc}
\hline Property & Unit & Lubricant A & Lubricant B & Lubricant C \\
\hline$\nu$ at $40^{\circ} C$ & $\mathrm{~mm}^{2} / \mathrm{s}$ & 13.78 & 47.3 & 99.0 \\
$\rho$ & $\mathrm{kg} / \mathrm{m}^{3}$ & 852 & 863 & 879 \\
$\alpha$ & $m m^{2} / \mathrm{N}$ & $21 \times 1 E-6$ & $21 \times 1 E-6$ & $21 \times 1 E-6$ \\
\hline
\end{tabular}

TABLE 3. Properties of lubricants.

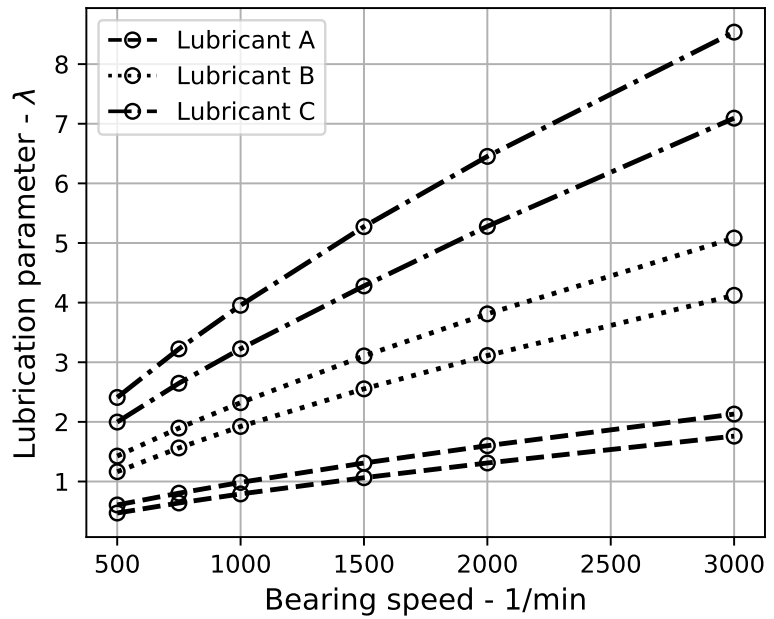

Figure 12. Effect of the external load and the viscosity on the lubrication coefficient.

- The bearing's external load is the least influencing factor. The substantial of its effect, however, increases at a higher speed and with the increasing viscosity of the lubricant. At low speeds, for the analysed case, the change of the load within the considered range doesn't produce a shift of the $\lambda$ to different lubrication regimes. The effect of the load is, in detail, depicted in the Figure 13 , where the load variation is captured in more steps for the Lubricant B.

- The bearing's running speed is obviously the factor driving the lubrication regime during the operation. For the current case and Lubricants B and C, each increase of speed by $500 \mathrm{rpm}$ means a transition between the lubrication regimes (e.g., from boundary through mixed to fully developed EHL). If evaluated by means of the $\lambda$ parameter, the low viscosity lubricant designated as A turned out to be inappropriate for the current case, because it does not provide a sufficient lubrication film to fully separate surfaces of the contact bodies. However, it might be useful at high speed applications, where the low viscosity is beneficial due to lower effects of the viscous friction.

- The lubricant's viscosity has a vast effect on the lubrication regime. The striking difference is obvious especially in low speeds, where the appropriate selection of lubricant's properties determines the separation of contact bodies by a lubricant and implicates a longer life.

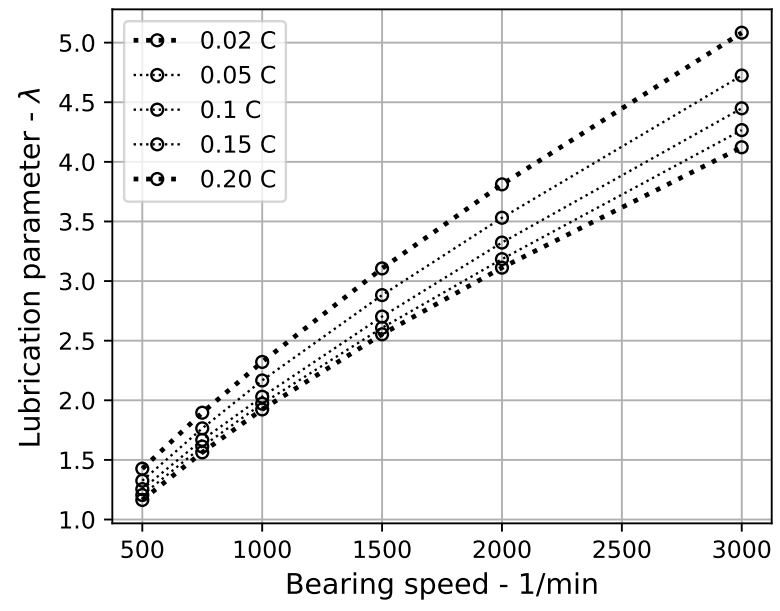

Figure 13. Detail of external load effect on change of the lubrication parameter $\lambda$ for Lubricant B per Table 3

The set of studies was performed to evaluate uncertainties raising from the estimation of lubricant properties and surface roughness after the running-in. In the first study, the viscosity-pressure coefficient $\alpha$ varied. It is not common that oil producers state the value in data-sheets, although in the literature [16], published values vary in a range from $18 / G P a$ to $22 / G P a$ for mineral oils with a medium to high VI and a temperature close to $40^{\circ} \mathrm{C}$. The result for Lubricant $\mathrm{B}$ and the load range $0.02 C-0.2 C$ is depicted in Figure 14

It is apparent that the difference can be distinguished especially in higher speeds. In the region of lower speeds, where the transition from one lubrication regime to the other one is observed, using any value will not introduce any significant bias to the study. If more detailed analysis would have been of interest, the value of viscosity-pressure coefficient should be further investigated to avoid any discrepancies. An example of the viscosity pressure coefficient determination method is in the [13.

The surface roughness has a significant impact on the formation of the lubrication film. During the running-in period, peaks of asperities are smoothed by plastic deformation and wear effects under contact, but the valleys remain. The gradual effect of the running-in on the surface roughness was experimentally studied by Wang et al. in [19], where for a contact with a zero value of the sliding-to-rolling ratio, the initial roughness $R a=0.5 \mu m$ was reduced by $30 \%$ at the end of the test. Because results for 


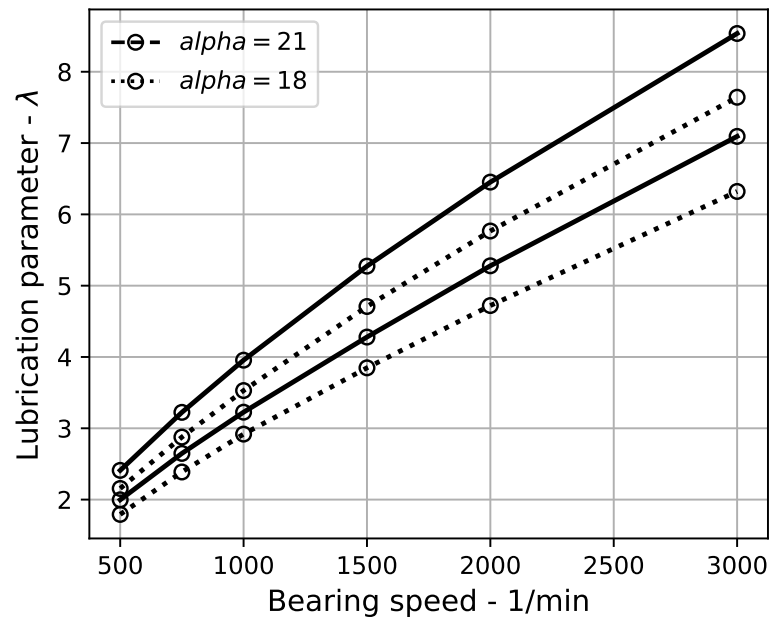

FIgURE 14. The sensitivity of $\lambda$ on variation of the viscosity-pressure coefficient $\alpha$. The dependency is calculated for Lubricant B per Table 3

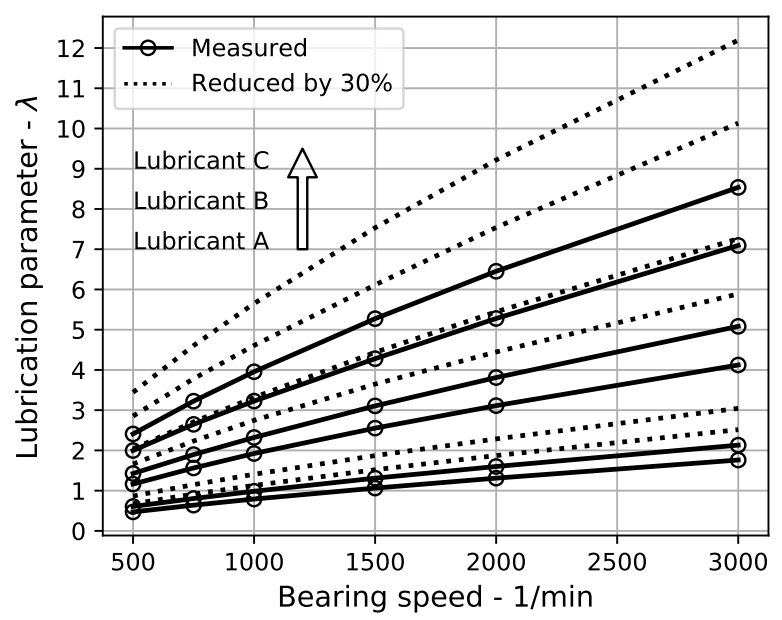

FIGURE 15 . The sensitivity of $\lambda$ on variation of the surface roughness.

roughness values corresponding to the measured in the current study were not found, the stated value was used to obtain its effect in the other study. The results are presented in the Figure 15 The pairs of solid lines represent the dependence of the measured roughness on the lubrication parameter for each lubricant $(\mathrm{A}, \mathrm{B}, \mathrm{C})$. The lower line represents the state under a low bearing load $0.02 C$ and the upper one the state under a high bearing load $0.2 C$. Dotted lines show the lubrication parameter for the reduced surface roughness.

The figure proves the importance of the surface finish in tribological contacts and the beneficial effect of running-in, if it is properly executed. The decrease of surface roughness during the period leads to a significant improvement of the lubrication parameter, it is especially obvious for the Lubricant B and $\mathrm{C}$ at low speeds, where it promotes the transition from the mixed to the EHL regime. The film formed by Lubricant A is of a similar thickness as the surface roughness, so when the current approach is employed, the improvement is not appearing to be much influential. Authors are, however, aware of that the $R q$ parameter used in the current study was calculated from the measured $R a$ value. The calculation assumes the normal distribution of the surface roughness, which was not verified. A significant relevancy improvement can be achieved by the direct assessment of the $R q$ parameter, or even better, employing the spatial parameter $S q$.

\section{Conclusions}

The study enabled an insight into the sensitivity of the roller bearing inner load distribution and lubrication regimes to the variation of the operating conditions. The described model takes the established ISO/TS 16281 [7] and extends it with the press fit and thermal gradient effects on the inner radial clearance. The model assumes a pure external radial load only and a low speed due neglected effects of centrifugal forces.

The results obtained by the bearing model for the measured bearing geometry indicate, that the internal radial clearance variation induced by the bearing assembly tolerance, the bearing press fit on the shaft and the temperature gradient influence the external load distribution over rolling elements. The significance of the effect follows the listing order, sorted from the most significant.

Following the bearing analysis, the numerical model of the lubricated line contact for the most loaded rolling element was afterwards applied to obtain a thickness of the EHL film and to assess the lubrication regime the bearing operates in.

The obtained outcomes show that the speed and the viscosity of the lubricant are the most influencing factors for the thickness of the EHL film. The impact of the surface roughness expressed by the $R a$ was included in the analysis by the $\lambda$ lubrication coefficient. The data show that the assumed $30 \%$ reduction of the surface roughness significantly improves the lubrication coefficient. The effect is most apparent for the lubricants with a higher viscosity (B and C).

The described analysis approach overcomes the main limitation of the extensively used ISO 281 [1], which assumes and covers information about the roller load distribution within one parameter - the dynamic capacity [4. It doesn't thus allow studying the effect of operating conditions on the load of individual elements.

The approach for the selection of the lubricant viscosity through the $\kappa$ and the $a_{i s o}$ described in the ISO 281 [1], neglects the problematics of the lubrication behind the coefficients, not allowing to include the effect of the surface roughness or more detailed analysis. The adoption of the EHL line contact model expressed by the Reynolds' equation was the enabler to study the thickness of the lubrication film with respect to operating conditions. 


\section{LIST OF SYMBOLS}

$i \quad$ Rolling element index

j Rolling element longitudinal coordinate index

$k$ Rolling element transversal coordinate index

$\psi \quad$ Rolling element position coordinate

$x \quad$ Rolling element transversal coordinate

$x^{\prime}$ Rolling element transversal auxiliary coordinate

$y$ Rolling element longitudinal coordinate

$F \quad$ Bearing radial load $[\mathrm{N}]$

$Q$ Roller load [N]

$K_{b}$ Load-deflection coefficient $\left[\mathrm{N} / \mathrm{mm}^{n}\right]$

$c$ Stiffness constant $\left[\mathrm{N} / \mathrm{mm}^{n}\right]$

$\delta$ Displacement $[\mathrm{mm}]$

$\delta_{u} \quad$ Bearing inner ring displacement [mm]

$n_{s} \quad$ No of roller lamina [-]

$f$ Load per roller lamina or general function $[\mathrm{N}]$

$R^{\prime} \quad$ Mutual contact radius: $1 / R^{\prime}=1 / R_{1}+1 / R_{2} \quad[\mathrm{~mm}]$

$E^{\prime} \quad$ Mutual Young's modulus $2 / E^{\prime}=\left(1-\xi_{1}^{2}\right) / E 1+(1-$ $\left.\xi_{2}^{2}\right) / E 2[\mathrm{~mm}]$

$\xi \quad$ Poisson's ratio $[\mathrm{mm}]$

$b \quad$ Width of hertz line contact area $[\mathrm{mm}]$

$p_{h} \quad$ Hertz contact pressure $\left[\mathrm{N} / \mathrm{mm}^{2}\right]$

$p$ Contact pressure $\left[\mathrm{N} / \mathrm{mm}^{2}\right]$

$n \quad$ Inner ring rotation speed $\left[\mathrm{min}^{-1}\right]$

$\omega_{\text {ir }}$ Inner ring rotation speed $[\mathrm{rad} / \mathrm{s}]$

us Roller-race entraining speed $[\mathrm{mm} / \mathrm{s}]$

$\Delta T$ Inner ring - Outer ring temperature gradient $\left[{ }^{\circ} \mathrm{C}\right]$

$\alpha_{T}$ Coefficient of linear thermal expansion $\left[{ }^{\circ} \mathrm{C}^{-1}\right]$

$C$ Bearing Dynamic Capacity [N]

$\left.a_{i s o}\right)$ Bearing life modifying coefficient [-]

$d_{i r} \quad$ Bearing inner race diameter $[\mathrm{mm}]$

$D_{\text {or }}$ Bearing outer race diameter $[\mathrm{mm}]$

$d_{s} \quad$ Bearing bore / shaft diameter [mm]

$D_{w}$ Roller diameter [mm]

$L_{w e}$ Roller length [mm]

$P C D$ Bearing pitch diameter $[\mathrm{mm}]$

$P_{d} \quad$ Bearing radial clearance $[\mathrm{mm}]$

$P_{d o} \quad$ Bearing measured radial clearance $[\mathrm{mm}]$

$P \quad$ Roller long. modification profile [mm]

$I \quad$ Interference fit between inner ring and shaft $[\mathrm{mm}]$

$R$ Roller long. modification radius [mm]

$A$ Roller long. modification start coordinate [mm]

es Upper limit deviation (shaft) [mm]

ei Lower limit deviation (shaft) [mm]

ES Upper limit deviation (hub) [mm]

EI Lower limit deviation (hub) [mm]

$h \quad$ Film thickness [m]

$h_{c} \quad$ Lubrication film central thickness [m]

$h_{0} \quad$ Lubrication film minimal thickness $[\mathrm{m}]$

$\nu$ Lubricants kinematic viscosity $\left[\mathrm{m}^{2} / \mathrm{s}\right]$

$\eta \quad$ Lubricants dynamic viscosity [Pas]

$\eta_{0} \quad$ Lubricants dynamic viscosity at ref. pressure [Pa s]

$\eta_{R}$ Rhoeland's reference viscosity: 6.315E-0.5 [Pa s]
$p_{R 1} \quad$ Reference pressure: $1.98 \mathrm{E} 8[\mathrm{~Pa}]$

$\alpha \quad$ Viscosity-pressure coefficient $[1 / \mathrm{Pa}]$

$\rho \quad$ Lubricant density $\left[\mathrm{kg} / \mathrm{m}^{3}\right]$

$\rho_{0} \quad$ Lubricant density at reference pressure $\left[\mathrm{kg} / \mathrm{m}^{3}\right]$

$p_{R 2} \quad$ Reference pressure: $5.9 \mathrm{E} 8[\mathrm{~Pa}]$

$\lambda$ Lubrication state parameter $[-]$

$R a$ Surface roughness - Arithmetic mean $[\mu \mathrm{m}]$

$R q$ Surface roughness - RMS value $[\mu \mathrm{m}]$

$S q$ Surface roughness - RMS value spatial $[\mu \mathrm{m}]$

\section{ACKNOWLEDGEMENTS}

This work was supported by the Grant Agency of the Czech Technical University in Prague, grant No. SGS18/127/OHK2/2T/12: Sensing of Rolling Bearings Lubrication Film by Surface Acoustic Leaky Waves Lambda.

\section{REFERENCES}

[1] ISO 281: 2007, Rolling bearings-dynamic loads ratings and rating life. International Organization for Standardization, Geneva, Switzerland 2007.

[2] H. Spikes. Basics of EHL for practical application. Lubrication Science 27(1):45-67, 2014. DOI: $10.1002 / \mathrm{ls} .1271$

[3] A. A. Lubrecht, C. H. Venner, F. Colin. Film thickness calculation in elasto-hydrodynamic lubricated line and elliptical contacts: The dowson, higginson, hamrock contribution. Proceedings of the Institution of Mechanical Engineers, Part J: Journal of Engineering Tribology 223(3):511-515, 2009. DOI:10.1243/13506501JET508

[4] T. A. Harris. Advanced Concepts of Bearing Technology, 5th Edition. CRC Press, 2006. DOI:10.1201/9781420006582

[5] J.-D. Wheeler, P. Vergne, N. Fillot, D. Philippon. On the relevance of analytical film thickness ehd equations for isothermal point contacts: Qualitative or quantitative predictions? Friction 4(4):369-379, 2016. DOI:10.1007/s40544-016-0133-7.

[6] T. A. Harris. Essential Concepts of Bearing Technology, 5th Edition. CRC Press, 2006. DOI:10.1201/9781420006599

[7] ISO/TS 16281: 2008, Rolling bearings-methods for calculating the modified reference rating life for universally loaded bearings. International Organization for Standardization, Geneva, Switzerland 2008.

[8] C. Venner. Multilevel solution of the EHL line and point contact problems. Ph.D. thesis, 1991.

[9] K. L. Johnson. Contact Mechanics. Cambridge University Press, 1985. DOI:10.1017/cbo9781139171731

[10] E. Bergseth, S. Björklund. Logarithmical crowning for spur gears. Journal of Mechanical Engineering 56(4):239-244, 2010.

[11] J. W. Eaton, D. Bateman, S. Hauberg, R. ik Wehbring. GNU Octave version 4.2.1 manual: a high-level interactiv e language for numerical computations, 2017.

[12] L. Nayak, K. Johnson. Pressure between elastic bodies having a slender area of contact and arbitrary profiles. International Journal of Mechanical Sciences 21(4):237-247, 1979. DOI:10.1016/0020-7403(79)90067-5 
[13] H. van Leeuwen. The determination of the pressure - viscosity coefficient of a lubricant through an accurate film thickness formula and accurate film thickness measurements. Proceedings of the Institution of Mechanical Engineers, Part J: Journal of Engineering Tribology 223(8):1143-1163, 2009. DOI:10.1243/13506501jet504.

[14] P. Yang. Multi-Grid Method, pp. 2333-2339. Springer US, Boston, MA, 2013. DOI:10.1007/978-0-387-92897-5_631.

[15] I. O. Aver'yanova, D. Y. Bogomolov, V. V. Poroshin. ISO 25178 standard for three-dimensional parametric assessment of surface texture. Russian Engineering Research 37(6):513-516, 2017. DOI:10.3103/S1068798X17060053
[16] Engineering tribology. In Engineering Tribology, p. i. Elsevier, 2013. DOI:10.1016/b978-0-12-397047-3.00018-7.

[17] ISO 5753-1: 2009, Rolling bearings - Internal clearance - Part 1: Radial internal clearance for radial bearings. International Organization for Standardization, Geneva, Switzerland 2009.

[18] ISO 492: 2014, Rolling bearings - Radial bearings Geometrical product specifications GPS and tolerance values. International Organization for Standardization, Geneva, Switzerland 2014.

[19] W. Wang, P. Wong, Z. Zhang. Experimental study of the real time change in surface roughness during running-in for PEHL contacts. Wear 244(1-2):140-146, 2000. DOI:10.1016/s0043-1648(00)00448-8 